Service social

\title{
Les itinérants de Montréal
}

\section{Pierre Simard}

Volume 39, numéro 2, 1990

Les problèmes sociaux

URI : https://id.erudit.org/iderudit/706477ar

DOI : https://doi.org/10.7202/706477ar

Aller au sommaire du numéro

\section{Éditeur(s)}

École de service social de l'Université Laval

\section{ISSN}

1708-1734 (numérique)

Découvrir la revue

\section{Citer cet article}

Simard, P. (1990). Les itinérants de Montréal. Service social, 39(2), 59-75.

https://doi.org/10.7202/706477ar

\section{Résumé de l'article}

Dans cet article, l'auteur décrit les conditions de vie des itinérants de Montréal. Ce qui l'amène à aborder divers aspects du phénomène de l'itinérance : le profil démographique des itinérants, les racines familiales, les expériences avec les institutions scolaires et sociosanitaires; les expériences de travail et surtout de chômage; la vie de couple et les séparations; le passage de la chambre à la rue, en passant par les refuges, tout en s'attachant à la fois aux conditions objectives de vie (la misère et la maladie) et aux conditions plus subjectives (la désorganisation psychosociale, la toxicomanie, la folie), pour finalement rappeler que « l'itinérant n'est pas né itinérant » et qu'en conséquence, il est important de comprendre le processus qui conduit vers la clochardise. 
Pierre Simard, travailleur social au

CLSC Centre-ville, Montréal.

\title{
Les itinérants de Montréal'
}

Pierre Simard

\author{
Un Canadien errant \\ banni de son foyer \\ parcourait en pleurant \\ un pays étranger.
}

L'itinérant pourrait reprendre à son compte les quatre lignes de cette complainte apprise sur les genoux de nos grands-parents. Ces derniers exprimaient ainsi leur mal du pays natal dont ils avaient été dépossédés et leur souffrance liée au sentiment d'aliénation qui en était résulté. En fredonnant leur nostalgie aux petits Canadiens français du temps, ils transmettaient à leurs rejetons devenus québécois les traces primitives de notre devise "Je me souviens".

La dépossession, I'aliénation et la souffrance font partie de I'univers de l'itinérant. Il survit quotidiennement dans un milieu à qui il est devenu étranger. II lutte à chaque jour pour repousser au loin, derrière un écran d'alcool, de drogue, de folie et de révolte, la tristesse de ses souvenirs, l'impuissance engendrée par ses échecs et le désespoir du pays devenu inaccessible.

L'itinérance n'est certes pas un phénomène récent. De la naissance à la mort, l'homo sapiens a connu cette condition durant la plus grande partie de son histoire. L'histoire ancienne et moderne est peuplée d'itinérants de tout acabit : le juif errant du temps biblique, les misérables du XVIII e siècle que nous a fait connaître Victor Hugo, et à notre époque, Calcutta et Rio de Janeiro. Les catastrophes de la nature, les récessions économiques, les guerres, les génocides ont tous provoqué l'éclosion d'itinérants, hier comme aujourd'hui. Par ailleurs, ce mode de vie n'a pas qu'une connotation négative dans la mythologie populaire; une aura de liberté semble encore entourer les déplacements mystérieux du gitan, du vagabond de grand chemin et du clochard-poète philosophant sur les bancs publics.

Les itinérants de Montréal échappent pourtant à ces dynamiques. Ils sont issus d'une société moderne, riche et en croissance 
économique; ils ne connaissent pas les désastres provoqués par les inondations, les tornades, la famine, la guerre, le génocide. Ils ont grandi dans une société qui donne accès à la santé, à l'éducation, à un revenu de soutien garanti. II n'est donc pas étonnant que leur situation soit souvent perçue comme le résultat d'un choix de vie, d'une recherche à courte vue de la liberté et du parasitisme. Pourtant, les fantasmes de liberté associés à leur mode de vie ont très peu de prise sur leur réalité de chaque jour.

Le porte-à-porte du mendiant est devenu quête agressive sur la rue Sainte-Catherine ou vol avec agression dans les bouches de métro; le poète-philosophe s'est transformé en psychotique délirant sur la place publique; le gîte du passant et la table accueillante ont été remplacés par les poubelles publiques qui servent d'auberge à la "bag-lady". À côté des images d'horreur venues d'ailleurs, étrangères autant qu'étranges, transmises par l'histoire ou par les caméras de télévision et montrant les enfants affamés d'Éthiopie, les " boatpeople " du Vietnam, les Noirs de Harlem à New York, les mouroirs de Calcutta, les bidonvilles d'Amérique du Sud, surgissent avec une égale intensité des loques humaines étendues sur le plancher de béton de Dernier Recours Montréal au cœur du centre-ville de la métropole, en 1990.

Un véritable problème social a maintenant pris place chez nous. II touche un grand nombre d'individus : 15636 itinérants ont été recensés à Montréal entre le $1^{\text {er }}$ mars 1988 et le 28 février 1989; il connaît une croissance explosive avec un taux de renouvellement se situant autour de $30 \%$, soit un ajout d'environ 4700 nouveaux itinérants par année ${ }^{2}$; il s'étend à des populations autrefois peu touchées, à savoir les femmes et les jeunes; il a un impact de plus en plus lourd sur l'environnement: insécurité croissante chez les résidents et les étudiants du centre-ville, impact économique négatif auprès des commerçants, etc. Le phénomène déborde les explications classiques reliées aux lacunes individuelles ou aux ratés épisodiques de l'économie; sa visibilité renvoie un constat quotidien d'échec à une société qui s'enorgueillit de posséder l'un des meilleurs systèmes de sécurité sociale au monde. Bref l'itinérance à Montréal ouvre un débat qui n'est pas prêt d'être résolu, ni relégué aux oubliettes.

C'est dans ce contexte qu'au milieu des années 1980, une recherche fut entreprise au CLSC Centre-ville de Montréal en vue de mieux cerner le profil de l'itinérant montréalais, vérifier dans quelle mesure cette "carrière " pouvait constituer un choix, saisir la dynamique qui conduit à la clochardise et entrevoir, si possible, des voies de sortie. Le CLSC avait besoin d'un état de la situation précis et fiable pour réorienter son intervention auprès de cette population en 
détresse. Cette étude se voulait par ailleurs une contribution importante à la réflexion des nombreux organismes privés et publics confrontés quotidiennement à la croissance accélérée du phénomène des sans-abri.

La recherche s'est déroulée dans quatre refuges qui accueillent plus de la moitié des itinérants de Montréal : l'Accueil Bonneau, la mission Old Brewery, la Maison du Père et la mission Saint Michael. Près de 40 personnes ont été associées, de près ou de loin, à cette entreprise scientifique, menée à l'aide d'un questionnaire comportant 212 questions et administré par des professionnels familiers avec les techniques d'entrevue et la problématique de l'itinérance. Les entrevues, d'une durée moyenne de 90 minutes, ont été effectuées entre 1984 et 1987 auprès de 101 hommes clochards et d'un groupe témoin de 89 assistés sociaux, de même âge, de même sexe et à la frontière de l'itinérance.

La très grande majorité des sujets ont répondu spontanément, sans détour, parfois avec beaucoup d'émotion. L'entrevue devenait une occasion de faire le bilan de toute une vie et de porter un regard sur le passé, le présent et le futur; pour la plupart, elle fut l'occasion privilégiée de combler le besoin de se raconter et d'être écouté. Le climat de la rencontre en disait plus long sur l'isolement et la solitude que toutes les questions composant l'entretien, clochards et assistés sociaux confondus.

\section{Le profil démographique}

Des questions multiples se posent sur le profil du clochard. Est-il jeune ou vieux ? Parle-t-il le gitan ? S'agit-il d'un célibataire endurci, étranger à l'amour, au couple, à la divinité ? Et surtout d'où vient-il ? Certains le croient issu essentiellement du macadam urbain. D'autres au contraire l'imaginent comme un inadapté déraciné de son milieu rural. Là où les rumeurs s'accordent, c'est dans l'image du clochard perçu comme un sous-produit de la pauvreté.

En fait, l'âge moyen des clochards se situe autour de 34-35 ans. Les trois quarts d'entre eux sont francophones, catholiques et croyants. Contrairement aux études, surtout américaines, qui le perçoivent comme décroché de toute institution sociale, l'itinérant montréalais conserve un lien étroit avec la religion. En effet, les deux tiers des itinérants, surtout les plus âgés, fréquentent encore l'église. Les deux tiers également sont célibataires, bien que $77 \%$ d'entre eux aient déjà vécu en couple. Par ailleurs, les trois quarts sont nés au Québec. L'autre quart provient surtout du Canada et, une faible partie, des pays extérieurs. Sur trois itinérants, deux sont issus de centres urbains et l'autre, du milieu rural. Fait remarquable, les deux 
tiers disent avoir vécu dans une famille de niveau économique moyen ou même aisé. On aurait donc tort de pointer du doigt le béton étouffant des centres urbains, l'isolement du rang rural ou encore la pauvreté des familles d'origine pour expliquer les sources du processus de la clochardise.

\section{Les racines familiales}

Tous les indicateurs pointent dans la même direction : le support familial a fait défaut au point de départ. De plus, le soutien subséquent des parents et de la fratrie a lui aussi été escamoté. Bien que l'assisté social n'ait pas, lui non plus, été gâté à ce niveau, presque tous les repères placent l'itinérant dans un état de carence beaucoup plus prononcé.

La famille d'origine de l'itinérant compte en moyenne six enfants. II n'y occupe aucun rang particulier. II a presque une chance sur deux ( $43 \%)$ d'avoir connu une famille éclatée. S'il a moins de 30 ans, la probabilité passe à deux sur trois. Au moment de la séparation de ses parents, il n'avait en moyenne que huit ans. Après l'éclatement, il a tout de même maintenu des relations minimales avec sa famille et, en premier lieu, avec sa mère.

Pourtant, deux itinérants sur cinq ont vécu moins de treize ans avec leur mère et, fait tragique, près de $20 \%$ ne l'ont pas aimée et ne se sont pas sentis aimés par elle. La brisure du premier noyau s'est répercutée bien sûr dans leur communication ultérieure. Les contacts actuels avec leur mère sont de fait peu nombreux et peu fréquents : $27 \%$ des itinérants ( $44 \%$ chez les jeunes) n'ont eu aucun contact avec elle au cours de la dernière année. Et quand le contact s'établit, il a été amorcé presque uniquement (92\%) par l'itinérant.

Le rapport avec le père n'est guère plus satisfaisant : $60 \%$ des itinérants de moins de 45 ans n'ont pas vécu avec lui au cours de leur adolescence, précisément au moment où ils auraient eu le plus besoin de son appui. Par ailleurs, un clochard sur trois n'a pas aimé son père et ne $s^{\prime}$ en est pas senti aimé. La courte durée de cohabitation, combinée à une relation affective fort écorchée, n'a pas permis au processus d'identification de se rendre à terme. II n'est donc pas étonnant de constater un sens de l'identité souvent fragile. Le rapport actuel entre père et fils est donc ténu : $39 \%$ n'ont eu aucun contact avec lui au cours de la dernière année comparativement à $11 \%$ seulement chez les assistés sociaux; et quand la communication s'établit, elle est initiée trois fois sur quatre par le fils.

On peut constater une meilleure relation avec la fratrie. La plupart ( $86 \%$ ) ont éprouvé un sentiment d'amour envers leurs frères et sœurs qui le leur ont bien rendu. Cette situation est peut-être liée à 
la solidarité vécue entre les enfants au travers des difficultés familiales. Ce sentiment, par contre, varie avec l'âge : plus le clochard est jeune, plus cette solidarité s'effrite. Bien que les contacts ne foisonnent pas, c'est malgré tout avec la fratrie qu'ils demeurent un peu plus nombreux, plus fréquents et plus égalitaires.

Si l'on jette un coup d'œil sur l'ensemble des rapports familiaux, les priorités s'établissent donc comme suit : les contacts, peu nombreux et peu fréquents, se font d'abord avec les frères et sœurs, ensuite la mère, puis le père; le rapport est de type plutôt égalitaire avec la fratrie, assez dépendant vis-à-vis le père et très dépendant face à la mère. Les caractéristiques de ce noyau familial éclaté vont laisser sur le clochard des traces perceptibles tout au long de son histoire ultérieure.

\section{Le passage à l'école}

Après l'expérience familiale de la petite enfance, l'école prend le relais et occupe, à ce titre, une place centrale dans le développement de l'enfant. Comment l'itinérant a-t-il vécu cette phase ? L'école lui a-t-elle permis de surmonter les difficultés initiales ou, au contraire, est-elle venue renforcer un sentiment d'échec qui avait commencé à se construire?

Sa scolarité moyenne est de 9,4 ans contre 10,8 ans chez les assistés sociaux. De même, son niveau d'aspiration éducationnelle est inférieur à celui des assistés sociaux. Ces deux éléments combinés présentent un facteur de risque : moins on est scolarisé, plus on a de chances de se retrouver clochard. En dépit de cela, il ne faut pas sousestimer leur potentiel de croissance quand on sait que le tiers d'entre eux ont mis fin à leurs études contre leur gré et que, après plusieurs années d'expérience de vie, un clochard sur deux aurait souhaité atteindre un niveau de scolarité plus poussé.

\section{Le voyage institutionnel}

Nous savions qu'avant d'entrer dans le réseau des refuges, un bon nombre d'itinérants avaient déjà eu un apprentissage à la vie institutionnelle. Nous ignorions dans quelle mesure ils avaient été touchés par ce processus et surtout si cet apprentissage avait été plus prononcé que chez les assistés sociaux. En réalité, le parcours institutionnel du clochard s'avère beaucoup plus lourd.

Un itinérant sur deux a connu la famille d'accueil ou le centre de réadaptation ou les deux; la moitié d'entre eux en ont gardé un mauvais souvenir. II n'est donc pas surprenant que les lacunes 
affectives expérimentées au cours de l'enfance et de l'adolescence aient produit chez ces jeunes adultes un niveau d'autonomie insuffisant pour voler de leurs propres ailes.

C'est ainsi qu'un grand nombre (21\%) sont entrés dans l'armée. Comme chacun sait, l'armée assure les besoins vitaux de ses membres en termes de nourriture, logement, vêtement, etc., en plus d'offrir un encadrement serré et une orientation qui laisse peu de place à l'initiative personnelle et au développement de l'autonomie. Et, fait particulier aux clochards, ils y ont séjourné beaucoup plus longtemps que les assistés sociaux : $62 \%$ de ces jeunes recrues y sont restées quatre ans ou plus, comparativement à $21 \%$ seulement chez les assistés sociaux.

Par ailleurs, un bon nombre ont eu des démêlés avec la justice : $28 \%$ ont consulté un avocat pour affaires criminelles contre $13 \%$ chez les assistés sociaux et cette tendance est encore plus prononcée chez les jeunes; plus de la moitié des itinérants (53\%) ont connu la prison, comparativement à $38 \%$ chez les assistés sociaux. Quant au souvenir qu'ils en gardent, il est négatif pour deux itinérants sur trois et, fait étonnant, positif pour le troisième.

La vie en refuge se situe donc en continuité avec l'histoire antécédente. Une fois sorti de la famille d'accueil, du centre de réadaptation, de l'armée ou de la prison, c'est tout naturellement vers le refuge que se dirigera l'itinérant.

\section{L'entrée au travail... et la sortie}

L'un des volets fondamentaux dans l'analyse de l'itinérance concerne la dimension économique. Tous ces hommes engagés dans la voie de la clochardise ont-ils déjà été insérés de façon active dans le système économique ? Si oui, de quelle façon ? Comment en sont-ils sortis pour se retrouver à la rue, perdus dans un refuge ou isolés dans une piaule ? Fait étonnant, presque tous ont une expérience de travail de quinze ans en moyenne; $59 \%$ sont entrés sur le marché du travail avant même d'atteindre 17 ans (67\% chez les jeunes). La majorité (59\%) ont un métier et l'ont exercé, pour la moitié d'entre eux, pendant plus de dix ans. Quant aux relations de travail, elles ont été bonnes pour la grande majorité, tant avec les patrons qu'avec les camarades de travail. Bref, le clochard a un passé de travailleur et a été très actif dans le circuit économique. Sur ce plan, son profil ne diffère pas de celui de l'assisté social.

Là où le tableau prend une couleur particulière, c'est dans l'expérience du chômage. S'il partage avec l'assisté social la mise à pied comme voie d'entrée au chômage, la maladie et le congédiement le touchent beaucoup plus souvent. Par ailleurs, un plus grand 
nombre $(61 \%)$ franchiront le cap des trois années de chômage. La perte d'emploi est un choc pour tout travailleur : elle peut mener au statut permanent d'assisté social si l'individu dispose de peu de ressources pour réintégrer le marché du travail; par ailleurs, elle sera catastrophique si elle entraîne de surcroît une désorganisation plus générale de la personne et conduit l'itinérant à la clochardise. C'est ce qui semble se passer quand on constate qu'une majorité de ces hommes amorcent la carrière de clochard en même temps ou quelques années après leur sortie du circuit économique.

Leur situation est-elle pour autant désespérée ? Un coup d'œil rapide sur l'image qu'ils projettent laisse peu d'espoir sur leur insertion éventuelle. Quand on leur pose la question, les trois quarts disent vouloir travailler et les deux tiers s'en sentent encore capables. Leur condition de vie n'a pas encore annihilé chez eux toute attente. Leur confrontation avec la rue les a tout au plus amenés à des aspirations plus modestes en termes de qualité d'emploi.

\section{L'incursion dans le couple... et ses suites}

Contrairement à certaines grandes villes américaines, et à New York en particulier, on ne voit pas encore à Montréal, sauf exception et de façon transitoire, le phénomène des familles itinérantes et des enfants sans abri. La clochardise se manifeste la plupart du temps sous les traits d'un individu adulte. Pourtant, au rythme où le climat social se détériore, ce phénomène ne saurait tarder à émerger. Bien que la grande majorité des clochards soient célibataires, on peut cependant se demander combien ont formé un couple, avec ou sans enfants. Et pour ceux qui ont eu des enfants, quel rapport s'est établi avec eux dans le passé et quelles traces de ces liens persistent encore aujourd'hui ?

La plupart $(77 \%)$ ont connu la vie de couple et, pour la moitié d'entre eux, durant une période de cinq ans ou plus. Ils sont maintenant tous séparés depuis plus de trois ans. Un clochard sur trois a eu des enfants qu'il a quittés la plupart du temps avant le début de leur adolescence. En ceci, il reprend à son compte le modèle qu'il a lui-même connu alors que son père l'avait quitté avant qu'il n'atteigne l'âge de 13 ans. Tous les clochards-pères (33 \%) disent avoir aimé leurs enfants et $62 \%$ d'entre eux maintiennent un contact minimal avec eux.

Tout compte fait, près de la moitié des itinérants ont coupé à peu près tout lien familial, tant avec leurs descendants qu'avec leurs ascendants. Pour les autres, le lien demeure plutôt fragile. Cet isolement ne sera certes pas d'un grand secours dans leur recherche 
de conditions de vie plus gratifiantes, ni aux assistés sociaux qui partagent globalement sur ce plan tes mêmes caractéristiques.

\section{De la chambre à la rue}

Retracer la piste du clochard dans le labyrinthe de son habitat est une entreprise malaisée. Les questions les plus directes comme les plus détournées, ajoutées aux réponses les plus franches et les plus élaborées, ne suffisent pas. Sa mémoire ne peut reproduire fidèlement le dédale des multiples lieux où l'a conduit son itinéraire, ni combler les trous provoqués par l'intoxication ou la perte occasionnelle du contact avec la réalité.

Compte tenu de ces limites, nous avons tout de même tracé à grands traits un parcours à peu près fidèle de ses résidences et de son territoire. Sur dix itinérants, on en retrouve trois en refuge, trois en chambre, un chez des amis, un à la rue et deux autres en prison, à l'urgence de l'hôpital, au poste de police, à l'unité de désintoxication. Par ailleurs, deux sur cinq ont connu la rue comme gîte au cours de la dernière année pendant une période moyenne d'un mois. Ce n'est pas Calcutta, mais non plus Outremont.

Ils ne demeurent pas longtemps à l'endroit où ils logent. En fait, la moitié occupent leur résidence actuelle depuis moins d'un mois. On peut le comprendre, car les nuits sont comptées pour ceux qui transitent en refuge; ils devront céder la place aux nouveaux arrivants qui attendent. Quant aux itinérants chambreurs, ils y resteront un peu plus longtemps, même si la plupart se réfugient dans les chambres les plus économiques et les plus délabrées du centre-ville et qui, de surcroît, se font de plus en plus rares. Malgré tout, leurs exigences demeurent très modestes, puisque $58 \%$ se disent satisfaits des conditions actuelles de leur habitat. Dans ce contexte, on ne sera pas surpris de constater que $80 \%$ des clochards ont déménagé au cours de l'année et que la moitié d'entre eux ont transporté leurs pénates au moins trois fois et souvent beaucoup plus. Les plus nomades se concentrent évidemment chez les jeunes et les itinérants vivant en refuge. Dans la moitié des cas, les départs ont été forcés. Ils ne vont d'ailleurs pas très loin : $72 \%$ sont des itinérants locaux qui circulent uniquement dans Montréal. Quelques-uns (14\%) sillonnent tout le Québec et $12 \%$ tout le Canada. De temps à autre (2\%), on croisera un clochard international.

Après ce mouvement incessant déjà décrit comme le phénomène de la porte tournante, on peut se demander s'il est encore possible pour les itinérants de nourrir des aspirations pour un mode d'habitat plus serein. En réalité, la presque totalité (93\%) désirent un toit bien à eux et les deux tiers d'entre eux choisiraient l'appartement comme 
lieu privilégié. Au-delà du désir, $71 \%$ s'attendent en fait à accéder à une adresse privée au cours de la prochaine année. Quant aux autres, c'est-à-dire un clochard sur quatre, leurs expectatives ont disparu : $19 \%$ n'ont aucune idée de leur avenir sur ce plan et $6 \%$ se voient en permanence en refuge. C'est surtout au sein de ce dernier groupe, qui rejoint tout de même $25 \%$ des itinérants, qu'on pourra repérer le clochard chronique, résigné à son sort, où même l'espoir n'a plus de gîte. À ce seuil, l'itinérance est sur le point d'être intégrée comme mode de vie et c'est à cette étape que s'offre à l'individu le " choix" de la clochardise. En tout cas, le choix est bien mince et plutôt angoissant quand, au bout du parcours, il ne reste plus que l'aliénation, la mort ou la résignation. Nous sommes bien loin du clochard philosophant sous les ponts de Paris ou du troubadour heureux et insouciant qui a choisi la chansonnette.

\section{En passant par le refuge}

La résidence de l'itinérant est, par définition, plutôt mobile. Le refuge pourtant y occupe une place importante; au moment de l'enquête, $53 \%$ des itinérants, et plus particulièrement les jeunes (81\%), y vivent. La durée du séjour n'excède généralement pas deux semaines. L'autre $47 \%$, présentement en chambre, se contente des autres services vitaux offerts par les centres de jour, soit la nourriture, le vêtement, un encadrement minimal et un certain contact humain. Au total, ils visitent 2,5 refuges par mois (3,2 chez les jeunes). Quand on se reporte à la première fois où l'itinérant a utilisé un refuge, on peut retracer son cheminement de la façon suivante : l'itinérance a débuté il y a moins d'un an pour $19 \%$ d'entre eux; c'est la phase de la crise. Un autre $35 \%$ est en période d'apprentissage depuis plus d'un an, mais moins de trois ans. L'autre groupe (29\%) s'enracine dans ce mode de vie depuis quatre ans et plus.

Quant au dernier groupe (17\%), il a déjà franchi le cap des dix années de clochardise; il ne changera probablement plus de " carrière ". La boucle se trouve ainsi bouclée. Le refuge constitue la dernière étape du parcours institutionnel amorcé au cours de l'enfance et poursuivi durant l'adolescence et au début de l'âge adulte. Le clochard a atteint la dernière frontière où il puisse encore poursuivre sa quête d'une ultime famille réparatrice. Au-delà se profilent l'ombre de l'asile psychiatrique et le vide de la morgue.

\section{Les revenus de survie}

Comme chacun sait, l'argent constitue le nerf de la guerre comme de la paix. De quelle source de revenu dispose l'itinérant pour 
survivre ? Où se procure-t-il l'argent nécessaire pour alimenter en plus sa toxicomanie ? Peut-il espérer avec le temps voir le bout du tunnel?

La plupart $(81 \%)$ vivent en fait de l'aide sociale. Par ailleurs, un nombre assez imposant (19\%), concentré massivement chez les jeunes, ne dispose d'aucun revenu officiel; et pour le tiers d'entre eux ( $37 \%)$, cette situation dure depuis plus de trois mois. Ce portrait ne tient pas compte évidemment des revenus d'appoint tirés occasionnellement du travail au noir, de la prostitution, du vol, de la vente de bouteilles vides ou de médicaments prescrits, du produit de la quête publique ou du trafic de la drogue. De plus, une forte proportion d'entre eux ont amorcé ce style de vie en même temps ou peu de temps après être devenus assistés sociaux. La précarité et l'insuffisance de revenus, ajoutées à d'autres facteurs de désorganisation, ouvrent davantage la porte d'entrée à l'itinérance, et cela est d'autant plus critique que se prolonge l'aide sociale.

\section{La désorganisation psychosociale}

Une histoire chargée, une personnalité aux assises fragiles, la perte d'emploi et la rupture du lien conjugal, tout cela contribue sûrement à faire basculer ces hommes dans le monde de la clochardise. À son tour, le mode de vie itinérant semble de nature à provoquer un état de désorganisation encore plus grand. De fait, au cours de sa vie, un clochard sur deux a consulté un travailleur social et un sur trois un psychologue. Ce taux est encore plus élevé chez les jeunes de moins de 30 ans. La visite au psychologue est plus récente : la désorganisation mentale semble suivre la désorganisation sociale. II consulte d'abord ce professionnel pour des problèmes d'ordre psychologique et beaucoup aussi pour sa toxicomanie. Quant au travailleur social, il recherche davantage son support à travers une relation d'aide suivie. Ce profil se retrouve aussi chez l'assisté social, mais de façon plus diffuse.

\section{L'éclosion des maladies}

Un contact, même superficiel, avec l'itinérance laisse supposer un état de santé assez pauvre. Une alimentation déficiente, la consommation excessive de produits toxiques multiples, un style de vie qui exclut tout traitement médical autre que ponctuel, l'utilisation inappropriée de la médication prescrite qui sert même parfois de monnaie d'échange, tous ces facteurs, et bien d'autres, ne peuvent qu'entraîner les itinérants sur le terrain de la maladie. 
Les indicateurs du niveau de santé utilisés reflètent une condition physique plutôt alarmante. Un clochard sur cinq (21\%) a été hospitalisé au cours de la dernière année et $37 \%$ d'entre eux l'ont été pour une période de plus d'un mois. Les motifs d'hospitalisation couvrent la maladie physique, la maladie mentale, les blessures et la toxicomanie. Les deux tiers ont consulté le médecin au cours de la dernière année pour des raisons à peu près similaires. Ce profil n'est pas différent de celui des assistés sociaux. Les clochards, cependant, se démarquent par deux particularités : d'abord un plus grand nombre $(91 \%$ contre $76 \%)$ ont déjà été hospitalisés, ce qui laisse supposer un état de santé plus précaire; ensuite, leurs attentes vis-àvis le médecin débordent largement les raisons strictement médicales. Ils recherchent davantage un support et un guide, en particulier pour les problèmes de toxicomanie ou d'ordre psychiatrique, problèmes pour lesquels le médecin se sent généralement moins réceptif ou moins bien préparé.

\section{L'évasion dans les toxicomanies}

La consommation d'alcool et de drogue est très largement répandue. Elle rejoint en effet $93 \%$ des itinérants. Les modèles de consommation ne sont pas pour autant identiques. Sur dix individus, on trouvera un abstinent qui ne touche à aucun produit toxique, quatre clochards qui consomment uniquement de I'alcool, quatre autres itinérants qui utilisent les drogues mineures, avec ou sans alcool, et enfin un dernier qui use aussi de drogues dures.

Qui dit consommateur ne dit pas nécessairement abuseur. Pourtant, les trois quarts se considèrent eux-mêmes toxicomanes et les raisons qu'ils apportent pour appuyer leur assertion ne laissent place à aucun doute : perte d'emploi, désintoxications répétées, séparation d'avec le conjoint, etc. Par ailleurs, contrairement à l'adage populaire, la sagesse ne semble croître ni avec l'âge ni avec une relative autonomie personnelle; en effet, le taux le plus élevé de toxicomanie se retrouve chez les 45 ans et plus ( $82 \%$ ) de même que chez les itinérants chambreurs $(85 \%)$. Bien que tous ces indicateurs soient largement présents parmi les assistés sociaux, leur concentration massive chez les itinérants place ces derniers dans les ligues majeures. Le mythe populaire du clochard robineux repose sur une réalité. La toxicomanie est directement liée au processus de clochardisation. Le tableau d'ensemble constitue tout un défi pour les intervenants qui osent encore le relever.

Et pourtant, une forte majorité $(77 \%)$ ont déjà tenté seuls de se sortir de ce problème et souhaitent toujours $(60 \%)$ y parvenir. Mieux encore, la moitié des toxicomanes ont déjà expérimenté une cure de 
désintoxication et des traitements appropriés. Quand on connaît la difficulté à surmonter une dépendance à l'alcool ou à la drogue, et compte tenu des problèmes multiples qui les confrontent quotidiennement, on est surpris de constater que le tiers des itinérants qui ont reçu l'aide nécessaire sont parvenus à stopper leur consommation durant un à trois ans. Ces derniers indicateurs ont de quoi réconforter les nombreux bénévoles qui se dévouent dans les refuges de Montréal et attireront peut-être l'attention des décideurs de politiques sociales qui relèguent les clochards au royaume de l'oubli et du désespoir.

\section{La fuite dans la folie}

Les nombreux contacts que nous avons eus avec les clochards au cours des années ne laissent aucun doute sur la place importante occupée par la maladie mentale dans ce milieu. Le phénomène est visible à l'œil nu. Pourtant ce style de vie, si marginal soit-il, ne traduit pas nécessairement la folie, c'est-à-dire la perte de contact avec la réalité extérieure ou la confusion grave du monde intérieur. Pour connaître l'étendue du problème et éviter les pièges de l'évaluation psychiatrique - nous ne voulions pas jouer aux apprentis sorciers nous avons retenu les références explicites à la maladie mentale exprimées par le clochard de Montréal au cours de son itinéraire dans le réseau des services de santé, et ses propres réflexions sur les causes l'ayant conduit à son style de vie.

L'investigation nous apprend que deux itinérants sur cinq (39\%) ont des problèmes de maladie mentale et que $30 \%$ des assistés sociaux partagent le même sort. Le faible écart entre les deux groupes écarte donc ce facteur comme cause explicative spécifique à l'itinérance. Cependant, la situation se retourne complètement quand on examine le cas des moins de 30 ans. En effet, $48 \%$ des jeunes clochards doivent se confronter à la folie comparativement à $18 \%$ seulement des jeunes assistés sociaux. On peut donc avancer avec assurance que folie et itinérance sont étroitement associées chez les jeunes clochards. La désinstitutionnalisation des hôpitaux psychiatriques, associée au manque de ressources alternatives, n'y est certainement pas étrangère.

\section{Retour sur le passé}

L'itinérant n'est pas né itinérant. Comme n'importe qui d'entre nous, il a vécu des expériences diverses qui l'ont conduit à ce mode de vie. Nous voulions saisir la dynamique, s'il en est une, de son 
cheminement. Pour y parvenir, il nous a paru important de connaître sa perception des événements les plus heureux et les plus traumatisants de sa vie et d'entendre son point de vue sur les causes l'ayant conduit à la clochardise.

L'événement le plus heureux et le plus malheureux est bien souvent relié aux liens affectifs ou amoureux. Pour un certain nombre cependant $(20 \%)$, l'entrée sur le marché du travail constitue leur meilleur souvenir. La plupart du temps, ces événements sont survenus à l'âge adulte. Toutefois, près de la moitié des clochards de moins de 45 ans ont vécu l'expérience la plus pénible au cours de leur enfance ou de leur adolescence. Le choc a donc été d'autant plus grand qu'il s'est produit à une période où les traumatismes laissent des traces plus profondes.

Pour expliquer leur style de vie actuel, ils invoquent surtout la toxicomanie (37\%), les difficultés reliées à la famille d'origine (27\%) et le manque de travail $(25 \%)$. Chez les assistés sociaux, on évoque surtout le manque d'argent ( $38 \%$ ), le manque de travail (33\%) et la maladie ou un accident ( $25 \%$ ). Les résultats de l'enquête nous présentent également de façon nette et précise les facteurs majeurs associés à la clochardise, soit les lacunes de la famille d'origine, les très nombreux problèmes de toxicomanie et le manque de travail. La perception du clochard sur les causes qui l'ont conduit à l'itinérance coïncide donc avec les résultats objectifs de notre investigation. La conscience qu'il a des problèmes est beaucoup plus poussée que ce que l'on serait porté à croire.

\section{Coup d'œil sur l'avenir}

Si le passé est garant de l'avenir, le futur des itinérants apparaît plutôt sombre. C'est du moins le point de vue de tout observateur extérieur. Et compte tenu du haut degré de marginalisation et d'impuissance vécues dans leur présent, on pourrait raisonnablement penser aussi que même l'espoir d'une amélioration de leur condition de vie n'existe plus, ou du moins a été fortement atténué au fil des ans. On s'attendrait en tout cas, sur ce plan, à une nette démarcation d'avec les assistés sociaux. Cette question n'est pas sans intérêt puisque l'espoir constitue une condition préliminaire essentielle à tout projet visant à modifier un style de vie qui baigne dans la désespérance. Nous avons donc exploré leurs perceptions de l'avenir en les interrogeant sur leurs espoirs vis-à-vis la qualité de vie, l'argent et l'amour.

Deux clochards sur trois n'aiment pas leur style de vie actuel et la grande majorité $(79 \%)$, incluant les plus âgés, ont espoir en une vie meilleure. Si le jeu à la Loto-Québec est un indicateur d'aspirations, 
on peut croire que la moitié recherchent encore un niveau de vie plus élevé, particulièrement chez les plus âgés. En outre, leur espérance de vie rejoint la moyenne nationale de l'âge du décès chez les hommes, soit autour de 74 ans. Ainsi donc, malgré toutes leurs carences et les difficultés sans nombre qu'ils affrontent, ces hommes conservent de façon étonnante des racines de vie. Sur tous ces plans, ils partagent le même profil que les assistés sociaux. Là où ils se distinguent, c'est au niveau des relations affectives. Même si les deux tiers déclarent avoir quelqu'un à aimer dans leur vie présente, près de la moitié $(45 \%)$ n'ont pas le sentiment d'être aimés.

\section{Le chemin vers la clochardise}

Cette recherche sur le clochard de Montréal repose sur 50000 données de base et près d'un million de chiffres. L'itinérant ne peut être réduit pour autant à une représentation statistique ou à une équation mathématique. II a un nom sinon une adresse. Derrière son profil se cache un visage dont les traits révèlent les traces d'un périple tumultueux. De cette multitude de renseignements s'échappent des bruits et des murmures qui courent en toutes directions, parfois les plus opposées. II faut pouvoir comprendre ces données, ces chiffres, ce qu'ils signifient.

En tout premier lieu, ils nous disent que le voyage de l'itinérant débute au sein même de sa famille dont le noyau central, les parents, est soumis à des pressions trop fortes qui aboutiront souvent à son éclatement. Une première fissure se produit alors au cœur des émotions et de l'estime de soi qui mettra beaucoup de temps, dans les meilleures conditions, à se colmater. Les familles ne se brisent pas toutes, mais quand cela se produit, ce sera généralement à peine quelques années après que l'enfant ait fait son entrée à l'école. Cet apprentissage scolaire va se dérouler pour un bon nombre (au cours de l'enfance ou au début de l'adolescence) dans un nid encore proche de la famille initiale, à savoir la famille d'accueil. Ce nid s'avérera pourtant plus instable encore et l'enfant devra apprendre, plus souvent qu'à son tour, à s'adapter à plusieurs familles substituts ou à expérimenter la froidure et l'anonymat du centre d'accueil ou de réadaptation.

Dans ce contexte, son périple scolaire ne fera pas long feu. II en sortira un peu plus tôt que les autres, à un moment justement où il n'a pas encore acquis les compétences intellectuelles et professionnelles suffisantes pour être autonome. II n'aura surtout pas encore appris à surmonter et à transformer les difficultés et les écueils inévitables d'un long parcours en apprentissage productif pour son développement ultérieur. Le dérapage académique viendra plutôt renforcer 
son sentiment d'échec en tant que personne. À cette phase, l'insécurité et l'angoisse augmentent et vont réclamer de façon de plus en plus impérieuse un antidote puissant qui puisse rendre la vie émotionnelle plus tolérable et faire taire ce sentiment d'échec qui crie toujours plus fort. C'est généralement au cours de cette période que l'apprenti clochard ira tester la soupape de la drogue et de l'alcool.

Curieusement, le pouvoir d'évasion va réussir à placer provisoirement l'insécurité de base sur une voie d'évitement et permettre au futur itinérant de faire le saut sur le marché du travail, puis de poser les premiers jalons d'une vie de couple. Un certain nombre iront même jusqu'à démarrer leur propre famille. D'autres opteront plutôt pour l'armée ou choisiront dans une certaine mesure la prison comme refuge substitut. Les liens au travail et au couple fourniront pour un temps l'encadrement nécessaire à l'amorce d'une vie adulte plus prometteuse. II ne sera pas exempt pour autant des tensions sociales qui assaillent tout le monde, ni immunisé contre les fluctuations de l'économie. Au contraire, sa fragilité le placera en tête du peloton de ceux qui connaîtront les premiers le congédiement et la séparation conjugale. Coupé du travail, de sa conjointe et de ses enfants, il se retrouvera isolé, avec de bien maigres ressources.

L'histoire suivra son cours de façon accélérée. Le départ de la maison et du monde du travail le mènera rapidement à un nouveau circuit caractérisé par "la porte tournante " à savoir la chambre, la rue, le refuge, la prison, la chambre... Ses nouveaux compagnons de voyage connaîtront des conditions de vie alternant entre l'isolement, la maladie, la toxicomanie et la folie. À l'occasion et plus souvent qu'autrement, on les retrouvera tous réunis. Cet itinéraire, plutôt simplifié, n'est pas suivi forcément par tous les clochards. Pour un certain nombre, le point de départ initial se situera de fait dans la famille éclatée. D'autres se joindront en cours de route, quand la drogue, la maladie mentale et l'inaccessibilité à l'emploi les auront dépouillés de leurs derniers mécanismes de défense. Le dernier groupe, plus résistant, viendra tout de même un peu plus tard rejoindre les pionniers au fur et à mesure que l'alcool, à action plus lente, et les déboires de la vie auront éliminé les dernières barrières.

\section{En guise de conclusion}

Ce récit pourra paraître exagéré dans son déroulement ou misérabiliste dans sa présentation. II n'en est rien. Ce que nous racontent les chiffres n'est qu'un faible reflet des confidences et des observations accumulées au cours des quinze dernières années. Leurs signaux ne constituent par ailleurs que la pointe du glacier. Pour un clochard couché sur un banc de parc, dix autres prendront sa place 
sous peu et un plus grand nombre encore semblent en voie de formation.

Les faits psychosociaux étudiés pourraient induire le lecteur à mettre la situation du clochard au compte de ses déficiences personnelles et familiales. En réalité, les faits ne disent pas tout et ne dévoilent surtout pas leur face cachée. Ils ne racontent pas pourquoi les familles éclatent de plus en plus, pourquoi un nombre sans cesse croissant de jeunes doivent recourir à la drogue, pourquoi l'emploi devient de plus en plus difficile d'accès, pourquoi le nombre de suicides chez les jeunes a monté en flèche depuis une dizaine d'années, ou encore pourquoi un nombre toujours grandissant de malades mentaux sont mis à la porte des hôpitaux psychiatriques et laissés seuls à la rue sans ressource alternative.

La réalité serait-elle trop sordide pour être affrontée, ou même nommée ? Ce serait I'histoire d'une autre recherche, au-delà du visible, et qui toucherait davantage les mécanismes de l'économie. On ne peut s'empêcher cependant d'y faire au moins allusion et de constater qu'il doit bien exister un lien quelconque entre la famille éclatée et l'orientation de la macroéconomie. Le retour en force du libéralisme économique axé sur la croissance économique sauvage n'y est certainement pas étranger. L'augmentation du produit national brut et la croissance mirobolante des entreprises n'entrainent pas pour autant la masse dans la prospérité. Au contraire, on assiste plutôt à la réapparition massive de la pauvreté, aux écarts de plus en plus prononcés entre les classes sociales, à une dégradation alarmante du milieu physique et atmosphérique et à une détérioration grave du tissu humain lui-même. Il faudra bien aller au-delà des équations et des cycles économiques pour concilier une économie de croissance avec la recherche première de la qualité de vie et la préservation du tissu social qui l'alimente à sa base même, soit la cellule familiale. L'itinérance en tout cas constitue une véritable bombe à retardement dont le mécanisme de mise à feu est déjà amorcé. Comme le mentionne Paul-André Comeau, rédacteur en chef du journal Le Devoir, dans sa préface à cette recherche :

Une société qui se flatte de voguer vers l'aisance peut-elle ignorer cette fraction de laissés-pour-compte ? [...] L'ampleur de ce drame, sa progression vertigineuse ne peuvent plus être minimisées. [...] L'intérêt de cette étude consiste précisément à situer le drame des sans-abri dans le glissement d'une société vers l'insouciance.

Les pouvoirs publics et nos élites sociales ne pourront plus se cantonner encore longtemps dans l'indifférence. Le problème exige une vraie riposte. L'espace manque ici pour examiner les réponses actuelles et explorer des voies de solutions plus adéquates, mais on 
peut prévoir dès maintenant que le chemin sera long, difficile et coûteux.

\begin{abstract}
Notes
1 Pour une analyse plus complète de la question, voir Le clochard de Montréal - une histoire à coucher dehors, rédigé par l'auteur de cet article et qui sera publié prochainement aux Éditions Saint-Martin. Le volume contient une présentation plus élaborée de la méthodologie utilisée, 152 tableaux statistiques commentés, un chapitre faisant ressortir les points communs entre itinérants et assistés sociaux, ainsi que les caractéristiques spécifiques au clochard, une photo de trois générations d'itinérants, des pistes de solution et dix récits authentiques de vie de clochards.

${ }^{2}$ CSSSRMM, Étude spéciale sur Dernier Recours Montréal, 1989, page ii.
\end{abstract}

\title{
Références
}

Coulombe, Dolores (1985). L'itinérance et le système de justice. Mémoire de maîtrise en criminologie, Université de Montréal, Montréal.

FourNIER, Louise et Cécile MERCIER (1989). Étude spéciale sur Dernier Recours Montréal. Montréal, CSSSRMM.

GAGNÉ, J. et H. DORVIL (1988). "L'itinérance, le regard sociologique ", Revue québécoise de psychologie, vol. 9, $\mathrm{n}^{\circ} 1:$ 63-78.

Lamontagne, Yves, Yvette Durand-Garceau, Suzanne Blais et Robert Élie (1987). La jeunesse québécoise et le phénomène des sans-abri. Québec : Presses de I'Université du Québec, Québec-Science Éditeur.

MCLAughuin, M.A. (1987). Les sans-abri au Canada, rapport sur l'enquête nationale. Conseil canadien de développement social, Ottawa/Montréal.

Ministère de la Main-d'œuvre et de la Sécurité du revenu (1988). Les sans-abri au Québec, étude exploratoire. Québec.

OUelLetTE, Françoise-Romaine (1989). Femmes sans toit ni voix. Québec : Les Publications du Québec.

PARAdIS, Marguerite (1990). Histoire de passion et de raison - jeunes et itinérantes. Montréal : Les éditions remue-ménage.

Ror, Shirley (1988). Seuls dans la rue. Montréal : Éditions Saint-Martin.

SIMARD, Pierre (1990). Le clochard de Montréal, une histoire à coucher dehors. Montréal : Éditions Saint-Martin (en cours d'édition).

Ville de Montréal (1987). Vers une politique municipale pour les sans-abri. Montréal, Comité des sans-abri. 